Proceedings of the 45th International School and Conference on the Physics of Semiconductors "Jaszowiec" 2016, Szczyrk

\title{
Transport Characteristics of Gated Core-Multishell Nanowires: Self-Consistent Approach
}

\author{
T. Palutkiewicz, M. Woøoszyn, P. Wójcik and B.J. Spisak* \\ AGH University of Science and Technology, Faculty of Physics and Applied Computer Science, \\ al. A. Mickiewicza 30, 30-059 Kraków, Poland
}

\begin{abstract}
The influence of the applied gate voltage on the coherent propagation of the conduction electrons through the InGaAs/InP core-multishell nanowires with the surrounding gate is considered. The solution of the threedimensional Schrödinger equation within the effective mass approximation is found using the adiabatic method. The electrostatic potential distribution generated by the all-around gate is determined from the self-consistent procedure applied to the Schrödinger-Poisson problem. The Landauer-Büttiker formalism and quantum transmission boundary method are applied to calculate the transport properties of the considered nanosystem.
\end{abstract}

DOI: 10.12693/APhysPolA.130.1190

PACS/topics: 72.80.Ey, 73.63.Nm, 85.35.-p

\section{Introduction}

Recent development of nanotechnology enables to fabricate the semiconductor nanowires (NW) with the coremultishell structure (CMS) for which the outer diameter is less than $30 \mathrm{~nm}[1]$. Such nanostructures find an application in the quantum electronics because of their transport properties which are determined by the quantum size effect and interference phenomena [2] and can be controlled by an external electric field generated by the electrostatic gates. This allows to construct the vertical transistor with all-around gate on the basis of the considered nanostructures [3]. However, the properties of such nanodevices strongly depend on materials and the geometric parameters, as we have shown in our previous report where we have investigated the influence of the gate voltage on the coherent propagation of the conduction electrons through the CMS-NW [4].

In the present report we generalize our previous results for a model including the self-consistent electrostatic potential distribution. For this purpose, we solve the threedimensional Schrödinger-Poisson problem and we determine the current-voltage characteristics of the CMS-NW via the Landauer-Büttiker theory. In contrast to the previous report [4], the radius of the core and thicknesses of shells are much smaller, which follows recent examples of experimental research [3].

\section{Method of calculations}

In the present study of a semiconductor CMS-NW we apply a theoretical model given in detail in our previous report [4], but with modified geometric parameters. The core and the outer shell of the nanowire are made of InGaAs, with the InP inner shell placed between them. The nanostructure is surrounded by an all-around gate placed close to the drain electrode. The $\mathrm{Hf}_{0.8} \mathrm{Al}_{0.2} \mathrm{O}$ insulator layer separates the gate and the wire.
The adiabatic approximation [5] is used to solve the 3D Schrödinger-Poisson problem. It is defined by the system of equations, which consists of the Schrödinger equation

$$
\left(-\frac{\hbar^{2}}{2 m^{*}} \nabla^{2}+U(\boldsymbol{r})\right) \psi(\boldsymbol{r})=E \psi(\boldsymbol{r})
$$

and the Poisson equation with the Dirichlet boundary condition for the electrostatic potential,

$$
\epsilon_{0} \nabla \cdot(\epsilon(\boldsymbol{r}) \nabla V(\boldsymbol{r}))=-\rho(\boldsymbol{r}),
$$

where $m^{*}$ is the effective mass of conduction electrons, $V(\boldsymbol{r})$ is the electrostatic potential and $\epsilon(r)$ is the positiondependent relative electric permittivity. The total potential energy of the conduction electrons consists of three terms

$$
U(\boldsymbol{r})=U_{\mathrm{C}}(\boldsymbol{r})+U_{\mathrm{B}}(\boldsymbol{r})+U_{\mathrm{e}}(\boldsymbol{r}),
$$

where $U_{\mathrm{C}}(r)$ is the hard-wall confinement energy, $U_{\mathrm{B}}(\boldsymbol{r})$ represents the profile of the conduction band bottom characterizing each layer of the nanowire, and $U_{\mathrm{e}}(\boldsymbol{r})=$ $-e V(\boldsymbol{r})$ is the potential energy resulting from the electron-electron interaction and the applied voltages. The charge density is given by the formula

$$
\rho(\boldsymbol{r})=-e\left(n(\boldsymbol{r})-N_{\mathrm{D}}(\boldsymbol{r})\right),
$$

where the carrier density $n(\boldsymbol{r})$ is calculated from the relation

$$
\begin{aligned}
& n(\boldsymbol{r})=\sum_{n} \int_{-\infty}^{\infty} \mathrm{d} E D(E)\left(f_{\mathrm{FD}}\left(E, \mu_{s}, T\right)\left|\psi_{n}^{s}(\boldsymbol{r} ; E)\right|^{2}\right. \\
& \left.\quad+f_{\mathrm{FD}}\left(E, \mu_{d}, T\right)\left|\psi_{n}^{d}(\boldsymbol{r} ; E)\right|^{2}\right)
\end{aligned}
$$

where

$$
f_{\mathrm{FD}}\left(E, E_{r}, T\right)=\left[1+\exp \left(\left(E-E_{r}\right) /\left(k_{\mathrm{B}} T\right)\right)\right]^{-1}
$$

is the Fermi-Dirac distribution at temperature $T$ with the electrochemical potential $\mu_{s}$ of the source, $\mu_{d}=$ $\mu_{s}-e V_{d s}$ of the drain, and $\psi_{n}^{s / d}(\boldsymbol{r} ; E)$ denote wave functions of electrons entering the device from source/drain electrodes, respectively. The total density of states $D(E)$ has the form 


$$
D(E)=\frac{\sqrt{E}}{(2 \pi)^{2}}\left(\frac{2 m^{*}}{\hbar}\right)^{\frac{3}{2}},
$$

and the donors distribution $N_{\mathrm{D}}(\boldsymbol{r})$ is given by the formula

$$
N_{\mathrm{D}}(\boldsymbol{r})= \begin{cases}N_{\mathrm{D}}, & \text { source and drain electrodes, } \\ 0, & \text { elsewhere }\end{cases}
$$

with $N_{\mathrm{D}}$ chosen to guarantee the charge neutrality of the device.

Self-consistent calculations used to determine the potential profile are based on the underrelaxation procedure

$$
V_{n+1}(\boldsymbol{r})=(1-\omega) V_{n}(\boldsymbol{r})+\omega V_{n+1}^{s}(\boldsymbol{r}),
$$

where the parameter $\omega$ has a value between 0.1 and 0.5 chosen to obtain the best possible performance, and $V_{n+1}^{s}(\boldsymbol{r})$ is obtained directly from the Poisson equation. Convergence of solution is assured by the condition $\Delta<\epsilon$ where $\Delta$ is the relative sum of square differences of the potential profiles between last two iterations,

$$
\Delta=\frac{\sum_{\boldsymbol{r}}\left(V_{n+1}(\boldsymbol{r})-V_{n}(\boldsymbol{r})\right)^{2}}{\sum_{\boldsymbol{r}} V_{n+1}^{2}(\boldsymbol{r})},
$$

and $\epsilon$ is the tolerance set to $10^{-5}$.

Calculations of every iteration are performed on a uniform computational grid in the following steps: (1) the Poisson equation is solved for the initial density of charge (which is set to zero), (2) the Schrödinger equation is solved for perpendicular 2D cross-sections of the wire, and (3) the wave functions along the $z$-axis are calculated for every energy in the considered range. These wave functions are used to determine the transmission coefficient which will be used for electric current calculations. They are also used to reconstruct the $3 \mathrm{D}$ wave functions required to find the density of charge which will be used in the next iteration of the self-consistent algorithm.

The analysis of the transport properties of the CMS nanowire is based on the electronic current formula [2]:

$$
\begin{aligned}
& I_{d}\left(V_{d s}, V_{g}, T\right)=\frac{2 e}{h} \sum_{n} \int_{-\infty}^{\infty} \mathrm{d} E \mathcal{T}_{n}\left(E ; V_{d s}, V_{g}\right) \\
& \quad \times\left(f_{\mathrm{FD}}\left(E, \mu_{s}, T\right)-f_{\mathrm{FD}}\left(E, \mu_{d}, T\right)\right),
\end{aligned}
$$

where $\mathcal{T}_{n}\left(E ; V_{d s}, V_{g}\right)$ is the transmission coefficient calculated from wave functions obtained from quantum transmission boundary method [6].

\section{Results and discussion}

We have used the parameters from Table I to obtain all the results presented below. For such choice of geometry and other parameters, the potential energy profile resulting from the self-consistent calculations was found in the form shown in Fig. 1 (for comparison, it also shows with dashed line the potential energy profile used in the calculations which do not include the self-consistent procedure). It demonstrates that the influence of the selfconsistent procedure is observed mainly within the core of the NW, which is a consequence of the largest charge density in this region.
TABLE I

Geometrical and material parameters of the single allaround gated core-shell nanowire.

\begin{tabular}{c|c|c}
\hline \hline wire length & $l_{w}=1200 \mathrm{~nm}$ & \\
gate length & $l_{g}=200 \mathrm{~nm}$ & \\
gate-drain distance & $l_{d}=50 \mathrm{~nm}$ & \\
source/drain electrode length & $l_{e}=100 \mathrm{~nm}$ & \\
core radius & $r_{c}=11 \mathrm{~nm}$ & \\
insulating shell thickness & $t_{i s}=4 \mathrm{~nm}$ & \\
oxide thickness & $t_{o x}=20 \mathrm{~nm}$ & \\
oxide relative permittivity & $\epsilon_{o x}=120$ & $\mathrm{TiO}_{2}[7]$ \\
core relative permittivity & $\epsilon_{c}=13$ & $\operatorname{In}_{0.7} \mathrm{Ga}_{0.3} \mathrm{As}[8]$ \\
shell relative permittivity & $\epsilon_{s}=10$ & $\operatorname{In}_{0.7} \mathrm{Ga}_{0.3} \mathrm{As}$ \\
temperature & $T=4 \mathrm{~K}$ & \\
Fermi energy & $E_{\mathrm{F}}=100 \mathrm{meV}$ & \\
effective mass & $m=0.04113$ & $\operatorname{In}_{0.7} \mathrm{Ga}_{0.3} \mathrm{As}$
\end{tabular}

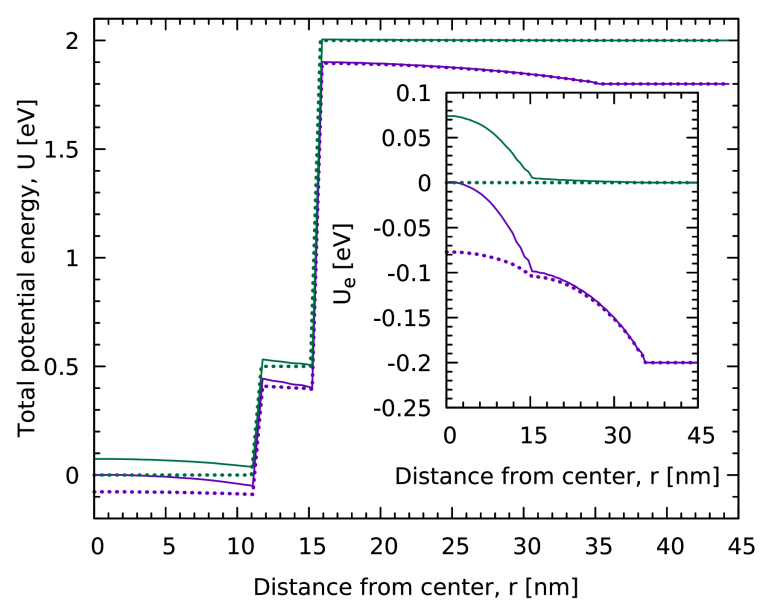

Fig. 1. The potential energy profile along the diameter of the nanowire in the middle of the gate (at $z=1200 \mathrm{~nm}$ ), for $V_{g}=0.2 \mathrm{~V}$ (dark, violet lines) and $V_{g}=0 \mathrm{~V}$ (light, green lines). Dashed line shows the potential energy profile used in the calculations without the self-consistent procedure and the inset presents the only $U_{\mathrm{e}}$ term of the potential energy.

The self-consistent solution of the Poisson-Schrödinger problem, which includes electric charge accumulated in the nanowire, leads to the current-voltage characteristics which is not symmetrical any more, as presented in Fig. 2.

Our calculations prove that for the negative values of $V_{d s}$, electric charge gathered in the potential energy well created by the gate near the drain electrode reduces the depth of the well. Therefore, it changes overall energy profile of the device, in a way which reduces the transport of electrons from the source to the drain. As a result of this, the absolute value of the negative electronic current increases. On the other hand, for the positive voltage $V_{d s}$, electrons gathered near the source electrode generate potential barrier which partially blocks transmission and reduces the output current.

Additionally, we have checked that for $V_{g}=0 \mathrm{~V}$ the solution is symmetric. It stems from the fact that the 


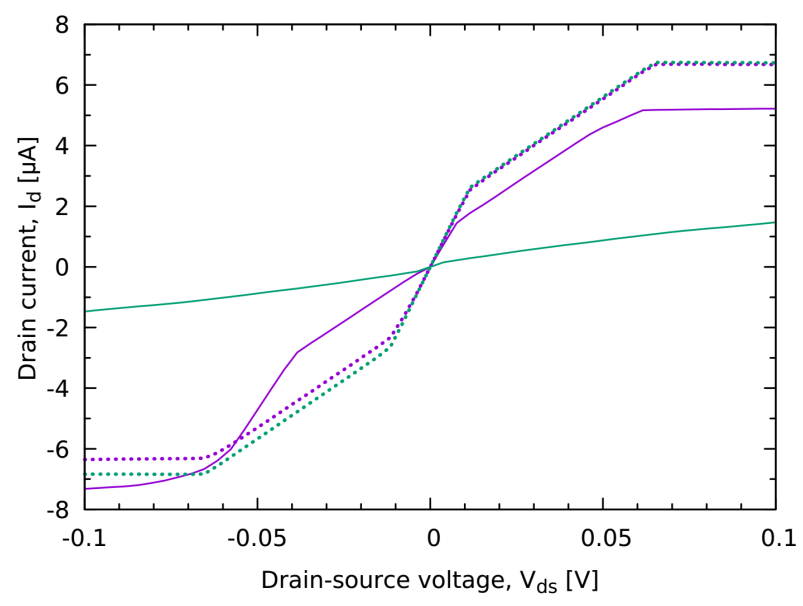

Fig. 2. The current-voltage characteristics calculated for the considered CMS-NW with the gate voltage $V_{g}=0.2 \mathrm{~V}$ (dark, violet lines) and $V_{g}=0 \mathrm{~V}$ (light, green lines). For comparison, the dashed line shows the result obtained when the self-consistent procedure was not used.

nanosystem contains no potential energy well in which electrons could concentrate. Simultaneously, the potential energy barriers generated by the electric charge gathered near the electrode of higher energy (from which the current flows) are also higher. In consequence, the electronic current is reduced when compared to the case with neglected accumulation of the electrons.

\section{Conclusions}

We have performed calculations of the current-voltage characteristics for the semiconductor nanowires with the core-multishell structure, applying the self-consistent procedure to determine the potential energy profile. The presented results indicate that the energy profile is strongly modified by the electric charge accumulated in the core region of the nanowire. In turn, it significantly affects the transmission coefficient and thereby we observe reduction of the electronic current through the considered nanosystem. The contribution of the accumulated electrons to the potential energy can either increase or decrease electric current, depending on the drain-source voltage. It is also affected by the position of the gate which is located asymmetrically in the proximity of the drain electrode in the considered case.

\section{Acknowledgments}

This work was partially supported by the funds of Polish Ministry of Science and Higher Education for 2016 and by PL-Grid Infrastructure. T.P. was supported by the scholarship of Krakow Smoluchowski Scientific Consortium from the funding for National Leading Research Centre by Ministry of Science and Higher Education (Poland).

\section{References}

[1] Y. Kohashi, S. Sakita, S. Hara, J. Motohisa, Appl. Phys. Express 6, 025502 (2013).

[2] D.K. Ferry, S.M. Goodnick, J. Bird, Transport in Nanostructures, Cambridge University Press, Cambridge 2009.

[3] X. Zhao, J.A. del Alamo, IEEE Electron. Dev. Lett. 35, 521 (2014).

[4] T. Palutkiewicz, M. Wołoszyn, J. Adamowski, P. Wójcik, B.J. Spisak, Acta Phys. Pol. A 129, A-111 (2015).

[5] M. Wołoszyn, B.J. Spisak, J. Adamowski, P. Wójcik, J. Phys. Condens. Matter 26, 325301 (2014).

[6] C.S. Lent, D.J. Kirkner, J. Appl. Phys. 67, 6353 (1990).

[7] S. Marinel, D.H. Choi, R. Heuguet, D. Agrawal, M. Lanagan, Ceram. Int. 39, 299 (2013).

[8] V. Gottschalch, Cryst. Res. Technol. 29, 702 (1994). 